\title{
Les enjeux de santé publique dans les pays en voie de développement
}

\section{Jean-Pierre Deschamps}

J.P. Deschamps: École de santé publique, Faculté de médecine, Université Henri-Poincaré-Nancy 1, 9, avenue de la Forêt-deHaye, BP 184, 54505 Vandœuvre-lèsNancy Cedex, France.

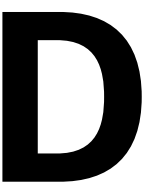
ans I'enthousiasme de la paix retrouvée, les trente médecins qui, en 1945 et 1946, à Paris, puis à New York, définissent les missions de la future Organisation Mondiale de la Santé (OMS), revendiquent des objectifs plus qu'ambitieux. Si nous ne sommes pas idéalistes aujourd'hui, disent-ils, quand le serat-on? On a beau jeu, aujourd'hui, de critiquer cet optimisme: pouvait-il en être autrement? Parmi les trois pionniers qui, en 1945 à San Francisco, dans les coulisses de l'Organisation des Nations Unies (ONU) naissante, avaient eu l'idée de l'OMS, l'un était brésilien, I'autre chinois [1]. La conférence de San Francisco va adopter à I'unanimité une motion sino-brésilienne permettant, en 1946, la signature de la charte de l'OMS. Ainsi, deux géants du "tiersmonde » sont acteurs, à ses origines, de la grande aventure internationale de la santé publique moderne.

\section{De Paris à Ottawa, 1946-1986:}

les impossibles prérequis de la santé

d'efforts véritacles, d'erreurs et de réalisations dérisoires, de projets généreux et de discours ambigus ont abouti à un cuisant échec.
«La possession du meilleur état de santé qu'il est capable d'atteindre, dit la Constitution de l'OMS, est un des droits fondamentaux de l'être humain. » Mais, il n'y a pas de bonne santé possible dans la pauvreté et I'indignité des conditions de vie. Quand I'OMS et I'UNICEF (United Nations Children's Found) rassemblent en 1986 la première Conférence internationale sur la promotion de la santé, le texte final, dit "Charte d'Ottawa», formule les "conditions préalables à la santé ». On mesure alors la distance qui sépare les principes de 1946 et les réalités de la fin du siècle. "Les conditions fondamentales de la santé sont la paix, un abri, l'éducation, la nourriture, un revenu, un écosystème stable, des ressources durables, la justice sociale et l'équité. L'amélioration de la santé exige d'être solidement ancrée sur ces prérequis fondamentaux."

Cette proclamation rappelle et explique la persistance de I'inégalité sociale de santé dans les pays industrialisés. Elle éclaire aussi violemment l'inégalité de santé entre les pays riches et les pays pauvres: quel pays du tiers-monde garantit à la majorité de ses habitants, trois des « conditions préalables à la santé »?

Dès lors, quels sont les enjeux de santé publique pour les pays en développement? Les politiques de santé doivent-elles attendre que les conditions préalables à leur efficacité soient assurées pour la plus grande part de leur population? Ou bien 
doivent-elles prendre à leur compte ces prérequis et en faire leurs objectifs au-delà de leurs missions traditionnelles? Le constat actuel, les échecs observés, appellent des stratégies nouvelles.

\section{L'état de santé dans les pays en développement}

De grands progrès ont été faits au cours de la seconde moitié du $X X^{e}$ siècle. L'espérance de vie a plus augmenté que dans les quatre millénaires précédents. Les politiques de vaccinations ont eu un effet incontestable. La variole a été éradiquée. Le programme élargi de vaccinations entrepris par I'OMS et I'UNICEF a permis une considérable régression de la poliomyélite, du tétanos néonatal, de la rougeole. Certaines endémies tropicales comme la dracunculose et l'onchocercose ont vu diminuer leur incidence et leur prévalence grâce à des politiques préventives ou curatives efficaces.

Deux éléments nuancent ce constat globalement favorable. Ces progrès ont essentiellement concerné les pays économiquement les plus avancés et ces indicateurs globaux masquent les inégalités d'état de santé d'un pays à l'autre et à l'intérieur des pays. Des maladies accessibles aux soins préventifs et curatifs gardent une fréquence considérable: la tuberculose reste responsable de près de 2 millions de décès annuels. Le paludisme, dont l'éradication avait été programmée pour 1970, provoque chaque année 300 millions de cas cliniques et un million de décès [2].

En résumant, l'état de santé du monde a fait des progrès mais l'écart entre les pays riches et les pays pauvres s'est accru. Cependant, les pays pauvres sont loin d'être homogènes. Le Sri Lanka, avec un PIB (produit intérieur brut) de 700 dollars par habitant, a une mortalité infantile de 16 pour 1000 , inférieure à celle de I'Argentine (22 pour $1000, \mathrm{PIB} / \mathrm{h}=$ 8030 dollars) et identique à celle des Émirats Arabes Unis (16 pour 1 000, $\mathrm{PIB} / \mathrm{h}=17400$ dollars). Le Costa Rica a un taux de mortalité infantile de 13 pour 1000; avec un PIB/h proche du sien (2 600 dollars), le Panama voisin a un taux de 23 pour 1000 , le Pérou un taux de 48 pour 1000 , la Thaï- lande un taux de 35 pour 1000 . Les pays $d^{\prime}$ Europe centrale et orientale ayant la meilleure espérance de vie à la naissance sont ceux où les inégalités de revenu sont les plus faibles [3]. Dans les pays les plus pauvres, en Afrique sous-saharienne, en Asie du Sud, en Amérique latine, le tableau reste dramatique. Le rapport 1999 de I'OMS sur la santé dans le monde rappelle que "plus d'un milliard de personnes vont aborder le $\mathrm{XXI}^{\mathrm{e}}$ siècle sans avoir profité de la révolution sanitaire: leur vie demeure brève et marquée par la maladie» [2].

La «transition épidémiologique» du tiers-monde constitue pour les pays concernés un "double fardeau ". Alors que les maladies infectieuses, les carences nutritionnelles et les complications de la grossesse et de l'accouchement sont loin d'avoir disparu, les affections dégénératives, les cancers et les accidents constituent une cause croissante de maladies et de décès. Le terme de transition n'est pas approprié pour les pays les plus vulnérables; il serait plus opportun de parler d'une addition de deux situations épidémiologiques. Les maladies non transmissibles constituent déjà $40 \%$ des années de vie perdues, corrigées de l'incapacité, dans les pays les plus pauvres [2].

A ce double fardeau s'ajoutent maintenant I'infection par le VIH, dont on sait la tragédie qu'elle représente notamment en Afrique et en Asie du Sud-Est, et le tabagisme, dont on estime qu'il tuera près de 500 millions de personnes, soit près de $10 \%$ de la population mondiale. Les pays à haut revenu, pour la plupart, se dotent de politiques de lutte contre I'usage du tabac, qui commencent à porter leurs fruits. Les industriels ont reporté leur effort de marketing dans l'immense marché que constituent les deux milliards de jeunes vivant dans les pays pauvres; une véritable hécatombe est délibérément planifiée.

Enfin, il faut évoquer l'inefficacité et la mauvaise qualité de la plupart des systèmes de santé. L'hygiène de la plupart des hôpitaux du tiers-monde est déplorable, les soins n'y sont souvent qu'une caricature de médecine, mettant en péril la santé des soignés et des soignants (par exemple, ceuxci ne disposent pas de gants leur permettant d'éviter les accidents d'exposition au sang lorsque les usagers ne peuvent les acheter). Les centres de santé de base fonctionnent au ralenti ; les personnels de santé n'y sont présents que 2 à 3 heures par jour; faute $d^{\prime} y$ être accueillis, et sachant qu'ils $n^{\prime} y$ trouveront pas de médicaments, les gens ne viennent pas. Au Laos, en 1999, des centres de santé étaient considérés comme fonctionnant bien s'ils recevaient au moins 7 patients par jour.

Les politiques du médicament sont peu efficaces. Les pays sont très dépendants des groupes pharmaceutiques internationaux: ceux-ci ont tout fait pour les empêcher de créer une industrie locale qui aurait pu fabriquer, à bas prix, des médicaments génériques en se limitant aux médicaments dits essentiels, et de valoriser les thérapeutiques traditionnelles. L'industrie occidentale n'a pas assez développé la fabrication de molécules ou de vaccins actifs sur les maladies tropicales, en raison de la non-solvabilité des populations concernées; elle s'est contentée de commercialiser les produits déjà mis sur le marché des pays riches.

Localement le stockage et la distribution des médicaments laissent beaucoup à désirer. L'usage de médicaments "anonymes», sortis de leur conditionnement, périmés, et de contrefaçons, est fréquent. Des médicaments distribués dans les hôpitaux ou envoyés de pays occidentaux par des émigrés sont vendus sur la plupart des marchés locaux.

\section{L'échec du modèle biomédical}

Les pays en développement, au moins ceux qui ont été colonisés, montrent la caricature des insuffisances d'une organisation des services de santé fondés sur une logique biomédicale. Ils rendent plus évident ce qu'un examen approfondi met aussi en lumière dans les pays industrialisés. Les conséquences en sont cependant différentes. Ici les progrès économiques et sociaux ont permis une élévation du niveau de santé telle que les systèmes de soins, en jouant à la marge, ont apporté une valeur ajoutée non négligeable malgré leurs défauts. Là, l'insuffisance de l'amélioration des conditions de vie a laissé aux seuls services de santé la responsabilité des progrès de l'état sanitaire. 
La tâche était titanesque: il aurait fallu s'y essayer avec des méthodes originales, spécifiquement étudiées pour répondre à cette situation, et non pas décalquer le modèle occidental d'organisation des soins et de formation des professionnels de santé.

Aucune analyse préalable des besoins et des demandes de la population n'a été menée. II aurait fallu la synergie de l'épidémiologie et des sciences humaines pour explorer non seulement l'état sanitaire, mais aussi les représentations, la part de la santé dans I'expression des cultures, le poids de l'organisation sociale, le rôle de l'affectivité et des structures familiales.

A quelques exceptions près, rien de tout cela n'a été fait. Du temps des colonies, et après I'accès à l'indépendance, on a bâti de grands hôpitaux dans les villes importantes. Si la construction était souvent financée par les pays occidentaux, les coûts de fonctionnement étaient d'emblée insupportables pour les pays receveurs. Ces structures rencontraient certes les demandes des classes dirigeantes, formées dans des écoles occidentales. A tant consommer pour les grands centres hospitaliers, il restait peu pour les services de base et les services intermédiaires. "Les systèmes de santé des pays en développement souffrent d'inadaptation et en particulier de surdéveloppement des structures hospitalières face à une pathologie qui relève avant tout d'actions extra-hospitalières", disait dans les années 1980 Michel Aurillac, ministre français de la Coopération (cité dans [4]). En dehors des églises et des organisations caritatives, les pays développés se sont peu intéressés à ces niveaux de soins. Les réseaux des dispensaires et des centres de santé, réduits à la portion congrue par l'indigence des finances publiques et la part prise par les hôpitaux, ont rarement bien fonctionné. Le personnel de santé, formé en occident ou à l'occidentale n'était pas préparé à exercer dans des structures de base; il faisait tout pour ne pas y être affecté ou ne pas y rester.

De ce point de vue, la Déclaration d'Alma Ata, en 1978, a été mal comprise. D'une part, elle mettait l'accent sur les soins de santé primaires comme éléments de base de systèmes de soins comportant un niveau secondaire - celui des districts - et un niveau tertiaire - celui des grandes villes et des régions - ; les soins de santé de base n'étaient acceptables que si ceux qui avaient une maladie grave pouvaient accéder aux autres niveaux, et cela n'était pas possible si les citadins et les personnes aisées recouraient directement à ceux-ci. L'existence de ces trois niveaux de soins exigeait une vision d'ensemble, une circulation des compétences et des informations de l'un à l'autre. D'autre part, les soins de santé primaires, par définition globaux et transversaux, ont vite été transformés, dans la tradition du modèle biomédical, en une série de programmes verticaux placés les uns à côté des autres, chacun avec ses financements, ses structures et ses personnels : programme élargi de vaccinations, programmes de lutte contre les diarrhées ou les infections respiratoires aiguës, programme de "santé reproductive », lutte contre la malnutrition, etc.

Une logique trop exclusivement fondée sur le modèle médical occidental a aussi freiné la mise en œuvre de mécanismes de participation communautaire, qui auraient permis la gestion des structures de base par des instances locales. L'émergence de celles-ci se heurtait à l'insuffisance d'une culture de démocratie, ainsi qu'à l'indigence des salaires, qui encourageait les professionnels de santé à s'approprier une grande part des recettes engendrées par cette gestion.

\section{Les grandes réformes des années 1980}

Il était devenu évident, dans les années 1970, que les États n'étaient pas en mesure de financer, en volume et en structure, les services publics qui constituaient l'essentiel de leurs systèmes de santé. Les politiques d'ajustement structurel imposées aux pays en développement par la Banque mondiale et le Fonds monétaire international pour leur permettre de rembourser leur dette en diminuant leurs dépenses publiques, ont contraint les pays pauvres à diminuer encore les ressources qu'ils affectaient à la santé et à l'éducation. L'autofinancement par- tiel des services de santé par les usagers est alors apparue comme une idée légitime, à condition qu'elle soit assortie de mesures d'accès aux soins pour les personnes les plus démunies, et que la qualité des services soit améliorée.

La réunion à Bamako en 1987, sous l'égide de I'UNICEF et de I'OMS, des ministres africains de la Santé, allait dans ce sens. Il s'agissait de répondre à la dégradation des services de santé des pays en développement, de restaurer la confiance des usagers, de relancer la politique des soins de santé primaires.

"L'initiative de Bamako» représentait un ensemble cohérent de mesures de réforme du secteur de la santé, articulé en huit principes: I'engagement des États à développer ce secteur, et en particulier à assurer des services disponibles à tous, la mise en œuvre d'une politique de médicaments essentiels, la décentralisation des procédures décisionnelles vers les districts, la gestion décentralisée et communautaire des structures de base, la participation des communautés (en fait des usagers) au paiement des soins, donc à l'approvisionnement en médicaments et au salaire du personnel, le soutien financier public aux soins de santé primaires, les mesures spécifiques d'accès aux soins pour les plus pauvres et la fixation d'objectifs intermédiaires et de critères d'évaluation.

Initialement limitée aux pays d'Afrique subsaharienne, I'initiative de Bamako a été étendue quelques années plus tard à des pays d'Asie (Yemen, Myanmar, Cambodge, Vietnam), d'Amérique latine (Pérou). Elle a reçu un large appui des organisations internationales et des instances de coopération gouvernementales et non gouvernementales.

Certains résultats sont encourageants, concernant notamment la couverture vaccinale et les soins prénatals. Des services de santé de base se sont développés et ont vu leur fréquentation s'accroître; des centrales d'achat et de distribution des médicaments essentiels génériques (MEG) ont vu le jour; du fait d'une meilleure gestion du prix du médicament, les dépenses de santé des familles ont pu diminuer, dans quelques pays, en dépit du paiement des consultations. Pourtant, tous les espoirs ne sont pas satisfaits. 
L'utilisation des MEG se heurte à de grandes résistances et à des pressions des firmes pharmaceutiques; la notion d'indigence, qui devait fonder la gratuité des soins, n'est pas claire; souvent ignorée, elle rend inéquitable I'utilisation des ressources.

Les plus grands obstacles concernent I'organisation communautaire et I'attitude des personnels de santé. La gestion des services de base par des structures communautaires se heurte à l'absence de statut juridique et au manque $d^{\prime}$ habitude du fonctionnement démocratique: les pouvoirs centraux et locaux n'y sont pas toujours favorables, la gestion financière s'accompagne d'abus de biens sociaux ou d'autres malversations; la lourdeur des contrôles administratifs est considérable. On appelle faussement «financement communautaire» ce qui n'est que le paiement individuel des prestations, et la réforme de Bamako apparaît pensée en fonction de structures rurales traditionnelles alors que la majorité de la population des pays en développement vit maintenant dans les villes et leurs banlieues. Enfin, I'absence de systèmes publics de protection sociale reste choquante [4].

Le problème des professionnels de santé prend l'allure d'une véritable crise. La formation initiale $n^{\prime}$ est pas adaptée, la formation continue absente; il n'existe pas de collaborations entre les personnels de districts et ceux des structures les plus périphériques, et ces dernières ne bénéficient d'aucune supervision; la partie des salaires versée par le service public est indigente, et servie avec retard; le complément de ressources permis par le paiement des usagers ne suffit pas à combler ces manques ; finalement, et surtout, le manque de motivation et l'absence de conscience d'un devoir éthique ne permettent pas l'amélioration espérée des performances.

Malgré des améliorations encourageantes dans quelques pays (Burkina Faso, Guinée, Mali, Malawi, Mauritanie, Nigeria, Sénégal), les réformes entamées à la fin des années 1980 ne suffisent pas à engager la « révolution sanitaire » appelée par l'OMS, encore moins à compenser les effets négatifs des politiques d'ajustement structurel sur le niveau des dépenses publiques sociales et d'éducation, ou les consé- quences sur l'économie des pays en développement des politiques néolibérales dans lesquelles s'inscrit l'initiative de Bamako.

\section{La santé et I'aide publique au développement; la coopération sanitaire internationale}

Le terme de «coopération» a été créé lors de la période de décolonisation, pour désigner des actions décidées et menées en commun par des pays du nord et du sud, devant se substituer aux opérations décidées par les seuls pays colonisateurs. Le terme était d'emblée irréaliste. II aurait été plus conforme à la réalité de garder le vocable «assistance technique».

L'aide publique au développement (APD) reste faible. En 1964, la conférence des Nations Unies pour le développement (CNUCED) demandait que I'APD représente $1 \%$ du PIB des pays développés, chiffre ramené ensuite à $0,7 \%$ : ce taux n'est atteint aujourd'hui que par quatre pays (Danemark, Norvège, Pays-Bas, Suède). La France consacre 0,45\% seulement de son PIB à I'APD, les États-Unis moins de $0,1 \%$ (mais en volume, leur part reste considérable). Les destinataires ne sont pas toujours les pays les plus pauvres (un tiers de I'APD française va aux DOM-TOM et le pays le plus aidé par les États-Unis est Israël). Dans I'après Seconde Guerre mondiale, le plan Marshall pour la reconstruction de l'Europe représentait 5 à 10 fois le volume de la coopération mondiale des années 1990, pour une population 5 à 10 fois moindre. Si I'on considère que les programmes de santé représentent $10 \%$ de l'APD, on mesure la modicité de la coopération sanitaire internationale, même si l'on y ajoute le secteur privé des organisations non gouvernementales.

La coopération comporte d'autres limites. Elle donne une priorité officielle («aide liée») ou officieuse aux intérêts des pays prestataires: soutien aux entreprises et aux exportations de biens et de services (matériel médical, médicaments, construction d'hôpitaux, utilisation des compagnies aériennes...), conditions avantageuses d'exploitation de ressources naturelles, pressions pour disposer de voix dans les instances internationales, avantages culturels (francophonie...).

Les programmes de coopération sont la plupart du temps imposés aux pays receveurs, sans possibilité pour eux de les négocier ou de les infléchir. Les programmes nationaux de lutte contre le sida des pays africains sont un exemple démonstratif d'importation de politiques de santé, non pensés avec les responsables des pays bénéficiaires, par l'OMS et la Coopération française [5].

Beaucoup de programmes de coopération internationale, multilatérale ou bilatérale, n'ont pas su aider à la mise en œuvre d'une véritable politique de santé, adaptée aux spécificités des pays en développement et ancrée dans la lutte contre la pauvreté. Le cas de la Coopération française est bien illustré par les rapports successifs de l'Observatoire permanent de la Coopération française [6] et dans l'analyse que fait cet organisme du rapport parlementaire élaboré par le député Y. Tavernier en 1998. La Coopération française $n^{\prime} a$ pas formulé d'objectifs explicites en matière de santé; $4 \%$ seulement de I'APD française va aux priorités du développement humain, dont rend compte un indicateur synthétique combinant l'espérance de vie à la naissance, le taux d'alphabétisation et le revenu moyen par habitant [7] (parmi les 25 pays les moins bien classés par I'indicateur de développement humain - IDH - figurent 23 pays $d^{\prime}$ Afrique subsaharienne largement «aidés » par la Coopération française depuis des décennies). Dès lors les priorités affichées concernent les services de santé; elles ne sont pas fondées sur l'analyse des besoins de santé des populations et envisagent d'emblée les réponses. D. Kerouedan et J. Brunet-Jailly font remarquer que la Coopération française ne s'est pas servie de l'expertise accumulée par ses assistants techniques [4]. Les politiques de coopération fondées sur la réforme des services font l'impasse sur les autres déterminants de la santé, économiques et sociaux. Elles singularisent la coopération sanitaire au lieu de l'intégrer dans une véritable politique d'aide au développement. Les vrais enjeux de la santé dans le tiers-monde sont en amont des services de santé. 


\section{Les priorités de l'amélioration de la santé dans le tiers-monde}

\section{Les conditions préalables...}

La première priorité est d'arrêter de mettre la charrue avant les bœufs. Le discours de la Banque Mondiale est erroné, qui répète à l'envi que "de bonnes pratiques de santé, de nutrition et de contrôle de la fertilité, et des services de santé efficaces sont les chaînons vulnérables dans la succession des événements qui permet aux nations de sortir du cercle infernal de la pauvreté, de la fécondité excessive, de la mauvaise santé et de la faible croissance économique, en lui substituant un cercle vertueux de productivité plus forte, de faible fertilité, de meilleure santé et d'augmentation des revenus »[8].

Il faut cesser de vouloir obtenir des personnes des «bonnes pratiques de santé »; au risque de paraître excessif, il faudrait aujourd'hui ne plus entreprendre de programmes $d^{\prime}$ " éducation sanitaire»: ceux-ci, en mettant l'accent sur le changement des comportements individuels, font implicitement peser sur les personnes la responsabilité des problèmes de santé, alors que les populations, écrasées par la pauvreté et la non-satisfaction de leurs besoins de base, n'ont pas la liberté de choisir leurs comportements. La scolarisation des filles a beaucoup plus d'effets sur le recours à la contraception, sur la fréquence des malnutritions ou la gravité des diarrhées, et finalement sur la mortalité infantile, que la masse des programmes verticaux d'éducation à la santé imposés à des populations qui n'ont même pas la possibilité de $s^{\prime} y$ soustraire. L'amélioration de la qualité des soins prénatals et des soins obstétricaux, et donc de la motivation et des compétences des personnels concernés, réduit plus sûrement la mortalité maternelle que l'information incantatoire des femmes sur la nécessité de la surveillance prénatale [9] à laquelle elles se soumettraient spontanément si elles étaient bien accueillies dans des services propres, bien équipés, animés par des professionnels bien formés.

La mise en œuvre de la charte d'Ottawa apparaît incontournable.
Toute politique de santé dans le tiersmonde doit $\mathrm{d}^{\prime}$ abord se fondre dans une politique plus globale répondant aux impératifs de développement humain définis par le Programme des Nations Unies pour le Développement: augmentation du revenu et réduction des inégalités sociales, augmentation du niveau de scolarisation, en particulier pour les filles et les jeunes femmes, mesures générales de politique économique allégeant la dette, fournissant aux pays en développement une aide juridique pour leur permettre d'avoir plus de poids à I'Organisation Mondiale du Commerce (OMC). Il faut sans cesse insister sur la nécessaire cohérence du développement: ce sont les mêmes qui doivent plaider et agir pour plus de justice économique et pour des programmes de santé efficaces.

L'environnement constitue une autre priorité à l'amélioration de la santé. La pénurie croissante d'eau douce, la pollution d'origine industrielle et agricole, la surexploitation forestière et des ressources minières appellent des systèmes de contrôle international pour faire diminuer leurs causes et des solutions technologiques coûteuses pour atténuer leurs conséquences. La gestion des villes, et plus particulièrement des mégalopoles du tiers-monde est un enjeu capital dont les solutions relèvent encore pour une part de la recherche technologique et en sciences humaines. Les appels à un développement « durable» (il est dommage de ne pas avoir traduit littéralement par «soutenable») ont, depuis 1972 et en particulier durant les années 1980, mis I'accent sur l'estimation du coût de I'altération de l'environnement liée à I'exploitation des matières premières, sur la nécessité de préserver le droit des générations futures à recevoir un patrimoine identique à celui que les précédentes ont trouvé, sur la répartition équitable des fruits du développement. Tout cela n'a guère dépassé le niveau des discours et $\mathrm{n}^{\prime}$ a pas modifié les modèles et les mécanismes décisionnels de l'économie mondiale.

Le dénominateur commun à ces problématiques relève pour une bonne part de la dérégulation économique et de la concentration des moyens industriels à une dimension vis-à-vis de laquelle le pouvoir des États appa- raît de plus en plus dérisoire. II faut se garder, lorsque I'on soumet le développement des politiques de santé à la condition d'une amélioration du revenu des personnes, de conclure que la croissance économique est le préalable à toute amélioration du bien-être. La croissance économique, mesurée à l'aune des agrégats nationaux, n'est pas le développement, mais sa conséquence.

Sur un autre plan, il est illusoire d'attendre une efficacité significative des politiques sanitaires sans changements structurels. Des alternatives commencent à se manifester. Face à I'uniformisation actuelle des modèles, elles ne peuvent résider que dans la diversité, les réalisations à petite échelle, au niveau de groupes philosophiques, religieux, socioprofessionnels, de communautés locales, de mouvements de défense de l'environnement, d'engagements féministes ou pour l'égalité des sexes. Ce n'est pas le lieu ici d'en faire l'inventaire. II faut seulement insister sur le fait que les responsables sociaux, les décideurs des politiques de coopération internationale, les responsables de santé publique ne peuvent les ignorer. II est encourageant de voir les rapports récents de la Banque Mondiale ou de l'OMS tenir des discours dans ce sens: il faut exercer une véritable veille auprès de ces organismes pour vérifier la conformité des discours et des pratiques, témoigner et plaider dans les réunions et les discussions, à quelque niveau qu'elles se tiennent... Garder le "pouvoir de s'indigner» est, dans le domaine social, I'un des moyens de prévention des violences institutionnelles: on peut transposer cet objectif à l'échelle mondiale; le sous-développement est une violence sociale et institutionnelle. Cessons de vouloir le résoudre, avec générosité et naïveté, par des programmes sectoriels ne remettant pas en cause les mécanismes qui le provoquent, l'entretiennent ou l'aggravent.

\section{Les politiques de santé}

La recherche sur les systèmes de santé, appliquée aux pays en développement, est pauvre en France, par comparaison avec les pays de langue anglaise; elle n'est pas considérée comme une priorité ou, pire, elle est 
parfois vue comme un luxe inutile dans un contexte où la gravité des problèmes rendrait l'action d'urgence nécessaire. Seuls, en France, les travaux d'économistes de la santé et les programmes récents de I'Institut de recherche pour le développement (IRD, ex-ORSTOM) échappent à cette faiblesse. On peut sans doute considérer que de celle-ci procède, par exemple, I'absence ou l'insuffisance des systèmes d'information sanitaire. Pour des indicateurs aussi fondamentaux que la mortalité infantile, I'OMS est obligée - sur la base de travaux anglo-américains de modélisation de recourir à des manœuvres de redressement des chiffres fournis par les pays [2]. Des travaux récents montrent l'intérêt d'approches nouvelles de l'information sanitaire, par exemple en Équateur [10]. Les systèmes à base épidémiologique, pour indispensables qu'ils soient, ne peuvent suffire à l'étude des besoins de santé des populations. Le recours aux sciences humaines est incontournable ; si l'ethnologie de la santé s'est bien épanouie dans les pays en développement - et ici les travaux français font autorité - le savoir qu'elle a créé a été peu utilisé de façon opérationnelle. La psychologie de la santé est pratiquement inconnue, alors que des travaux récemment publiés montrent le rôle capital des facteurs affectifs, par exemple dans la survenue des malnutritions [11-13].

Un second enjeu concerne la formation des professionnels de santé. Là encore, on manque d'études sur les profils de compétences et les besoins de formations spécifiques au tiersmonde. Les mécanismes actuels de sélection et de formation des enseignants rendent inéluctable la reproduction des schémas de formation des pays du nord. Pour ce qui concerne les médecins, les facultés " orientées vers la communauté » sont encore rares; I'exemple du Centre universitaire de formation des professionnels de santé, à Ho Chi MinhVille (Vietnam), reste peu suivi. La formation continue reste à créer. Elle pourrait être un moyen précieux de correction des erreurs de la formation initiale, à condition qu'elle ne soit pas confiée, pour l'instant, aux mêmes institutions. Là où elle existe, elle est souvent limitée à de brèves sessions, organisées dans la capitale, très médicocentrées, non accessibles aux personnels du terrain. Des modalités alternatives sont à trouver, par exemple des formations à distance, sur support traditionnel ou par Internet, tel que cela est actuellement essayé au Maroc, sur la base de l'expérience acquise par l'École de santé publique de Nancy.

L'éthique professionnelle constitue un élément qu'il serait urgent $d^{\prime}$ inclure dans les programmes de formation. La médecine occidentale procède de valeurs codifiées depuis plus de deux millénaires, complétées par des références judéo-chrétiennes et constamment actualisées. II n'existe pas une telle base culturelle pour la médecine des pays en développement, où les valeurs inscrites dans les médecines traditionnelles ne suffisent pas à fonder une éthique et une déontologie adaptées à la médecine moderne (mais elles peuvent y contribuer). Une autre forme d'éthique est à développer; elle concerne les dirigeants des pays du tiers-monde et les industriels qui y font commerce. Il s'agit de définir des règles de bonne conduite commerciale à propos des produits ayant une influence sur la santé. L'OMS et I'UNICEF ont pu faire accepter un code de commercialisation des aliments infantiles; ce code est encore loin d'être appliqué, mais il existe. Les industriels du médicament et du tabac sont directement concernés. Dans le contexte politicoéconomique mondial du moment, cela relève sans doute de l'utopie. II serait alors souhaitable qu'au moins soit mis en place par les pays - associés pour la circonstance - des systèmes de contrôle efficace, et que des réformes des procédures décisionnelles des institutions de Bretton Wood et de I'OMC donnent plus de poids aux pays en développement. Autre enjeu fondamental, I'organisation des services de santé doit être recentrée sur le niveau géopolitique essentiel que constitue le district (ou son équivalent). Vouloir développer les services de santé de base sans asseoir des structures de référence serait irréaliste; ces dernières ne peuvent être les grands hôpitaux alors que le niveau intermédiaire peut, sans négliger les relations avec elles, être proche des soins de santé primaires, et leur donner tout son sens. La gestion des structures de base et des structures intermédiaires ne peut être, malgré les difficultés, autre que communautaire. Mais il faut cesser les incantations à la "santé communautaire»: il ne peut y avoir de démarche communautaire de santé hors d'un processus de développement social communautaire. Une nouvelle génération de mouvements communautaires émerge, plus réalistes que dans les années 1970. Par exemple, les mutuelles du Mali, à propos desquelles un dirigeant malien disait en 1993 que "l'émergence de structures communautaires de soins semble la seule option de développement actuellement envisageable» (revue dans [14, 15]). Bien que plus exclusivement centrées sur la santé, les formations sanitaires urbaines à base communautaire (FSU.com) en Côte d'Ivoire ont l'intérêt d'adapter au contexte urbain ce qui a été pensé initialement pour les campagnes.

Un autre enjeu, pour conclure, est de considérer que les stratégies mises en œuvre au sud serviront aussi au nord. Les pays industrialisés ont beaucoup à apprendre des pays en développement...

\section{Remerciements}

L'auteur remercie pour leurs précieuses informations et remarques les Docteurs Hadissa Tapsoba, Dominique Kerouedan, Françoise et Michel Marquis, et le Professeur Jean-Claude Fritz. II exprime aussi sa reconnaissance, pour leur lecture critique et leurs conseils, aux Professeurs Anne-Marie Moulin et Gérard de Pouvourville.

\section{RÉFÉRENCES}

1. Deschamps JP. De Paris (1946) à Ottawa (1986): 40 ans de promotion de la santé. La Santé de l'Homme 1996; 325: VI-X.

2. Smith GD, Egger $M$. Understand it all health, meta-theories, and mortality trends. Br Med J 1996; 313: 1584-5.

3. Organisation Sociale de la Santé. Pour un réel changement: rapport sur la santé dans le monde 1999. Genève: OMS, 1999.

4. Kerouedan D, Brunet-Jailly J. La France at-elle une stratégie de coopération en matière de santé avec l'Afrique et Madagascar. Paris, Observatoire permanent de la Coopération française, 2000 (sous presse). 


\section{RÉFÉRENCES}

5. Kerouedan D, Eboko F. Politiques publiques du sida en Afrique. Centre d'Étude d'Afrique Noire. Travaux et documents. Paris: CNRS, 1999; 61-62: 73 p.

6. Floury B. Le discours de la coopération française en matière de santé depuis les indépendances. Observatoire permanent de la Coopération française, rapport 1998. Paris: Karthala, 1998:161-79.

7. Observatoire permanent de la Coopération française. Aide publique au développement: la réforme esquivée. Paris: Observatoire permanent de la Coopération française, 1999, document dactylographié, 5 p.

8. Banque Mondiale. Health, nutrition and population. Washington: The World Bank Group, 1997.

9. Prual A. Grossesse et accouchement en Afrique de l'Ouest. Santé Publique 1999; $11: 155-91$.

10. Briand S. Changement de paradigme dans I'évaluation des systèmes de surveillance épidémiologique. Ruptures (Montréal) 2000 (sous presse).
11. Geber M. L'enfant africain dans un monde en changement. Les champs de la santé. Paris: PUF, 1998.

12. Geber M. L'abandonnisme en Afrique sud-saharienne. Les champs de la santé. Paris: PUF, 1999.

13. Roger-Petitjean M. Soins et nutrition des enfants en milieu urbain africain. Paroles de mères. Paris: L'Harmattan, 1999.

14. Unger JP. Des solutions de rechange existent en Afrique. Le Monde Diplomatique, novembre 1993: 24-5.

15. Thomazeau AM. La voie de la mutualité. Le Monde Diplomatique, novembre 1993: 24-5

\section{TIRÉS À PART}

J.P. Deschamps.

\section{$\mathbf{m} / \mathbf{S} 2000$ \\ Summary}

Public health stake

in developing countries

«Health for all in the year 2000!» When the World Health Assembly issued this rallying cry in 1977 it did not mean the "state of complete physical, mental and social wellbeing " evoked in 1946 but «a level of health... permitting a socially and economically productive life». In the year 2000 the objective is as remote as it was twenty years ago - if not still more so. The health status of the poorest nations remains catastrophic. Health services are inaccessible to the majority of citizens and where they do exist their quality is often mediocre. Decades of real effort, of errors and derisory outcomes, of generous projects and ambiguous statements have terminated in painful failure.

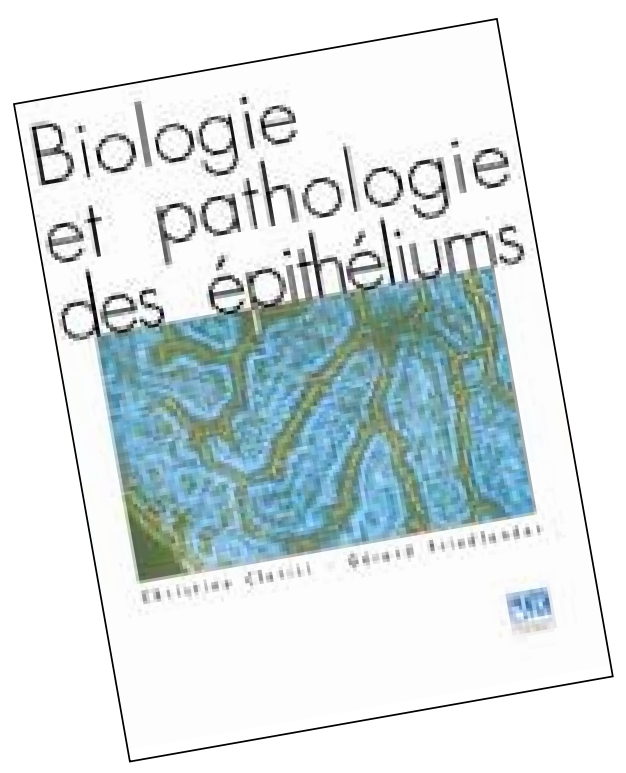

